\title{
Preliminary evaluation of exome sequencing to identify genetic markers of susceptibility to tuberculosis disease
}

\author{
Carla Duncan ${ }^{1}$, Frances Jamieson ${ }^{1,2}$ and Carolina Mehaffy ${ }^{3 *}$
}

\begin{abstract}
Background: Recent studies have shown that certain human genetic polymorphisms could be associated with susceptibility to tuberculosis (TB) infection and disease. Advances in next generation sequencing include the ability to rapidly sequence the entire human exome. These new technologies can be exploited to identify new associations of human genetic polymorphisms and TB infection and disease. In this preliminary study we compared two different strategies for sequencing of the human exome in a small sample set consisting of three individuals with a history of TB disease and two individuals with latent TB infection.
\end{abstract}

Findings: Sequencing of the entire exome of the five participants using Agilent SureSelect kit resulted in the identification of 1611 single nucleotide polymorphisms (SNPs) that were only present in the individuals with a history of active TB but not in the latent TB cases. Alternatively, sequencing of 4000 target genes available in the TruSight kit resulted in identification of 182 SNPs only present in the active TB cases and not in the latent TB participants. The overlap of the two kits was 112 SNPS.

Conclusions: Even though this pilot study was restricted to a small number of participants, we demonstrated the feasibility of using exome sequencing technologies to mine potential genetic associations of susceptibility to TB disease and presented a number of potential targets that can be further explore in larger research trials.

Keywords: Exome sequencing, Single nucleotide polymorphisms, Tuberculosis

\section{Findings}

\section{Rationale}

Recent studies have shown that certain human genetic polymorphisms could be associated with susceptibility to TB [1-10]. Many of the tested genes identified todate are involved directly or indirectly with immune and inflammation mechanisms [4-10]. However, it is possible that other polymorphisms outside of these target genes may be involved in susceptibility to TB. Recent advances in sequencing technologies and tools have made it possible to rapidly sequence the entire coding region of the human genome (exome) and allowing the identification of novel polymorphisms associated with a particular

\footnotetext{
*Correspondence: Carolina.mehaffy@colostate.edu

${ }^{3}$ Department of Microbiology, Immunology and Pathology, Colorado State University, 1619 Campus Delivery, Fort Collins, CO 80523-1601, USA Full list of author information is available at the end of the article
}

disease, disorder or condition [11-14]. Exome sequencing has been exploited to evaluate gene variants associated with certain cancers, however, its use in the study of susceptibility to infectious diseases is still limited [15, 16]. Tuberculosis infection and disease has a long history of association with the human race $[17,18]$ and given its intricate relationship with the host, it is not surprising that certain genes and gene variants or mutations have been thought to be involved in the ability of the host to fight the infection and/or halt its progression to disease.

\section{Goal}

The main focus of this pilot study was to evaluate the utility of two different kits for exome sequencing as a means to identify novel polymorphisms associated with susceptibility to TB. In Toronto, Canada, the largest clusters of active TB transmission are associated with the under 
housed or homeless population [19-21] and therefore another goal of the study was to evaluate the feasibility to recruit participants in this group, including individuals with past history of TB disease as well as individuals with a record of $\mathrm{TB}$ infection.

\section{Ethics, consent and permission}

Ethics approval for this study was granted by the institutional Ethics Research Board at Public Health Ontario, file number 2013-038. All participants received and signed an informed consent to voluntarily participate in this study.

\section{Methods}

After ethics approval was obtained, we conducted 1 month of recruitment at one of Toronto's inner city shelters. In total we recruited nine individuals. Three of these had a positive history of active TB disease for which the genotyping data indicated their infection was due to either one of the four $M$. tuberculosis strains previously associated with this population $[19,22]$. The remaining six individuals were recruited as potential candidates for the latent TB control group, however, only two of them had a record of a positive TB skin test indicating TB latent infection. The four individuals with no proven record of TB infection were not enrolled in the study. Blood samples were obtained from all participants and used to extract gDNA using the Qiagen QIAamp DNA blood mini kit as per the manufacture's protocol. Two different sequencing kits were evaluated, Agilent SureSelect $^{\mathrm{XT}}$ Target Enrichment System-Human All Exon $\mathrm{V} 5$ + UTRs and Illumina TruSight ${ }^{\mathrm{TM}}$ One. Illumina TruSight $^{\mathrm{TM}}$ One targets 4813 genes, including several immune related genes previously shown to be involved in TB infection and or host-pathogen interactions. gDNA from all participants was used to prepare the libraries for each kit as recommended by the manufacturer. SureSelect libraries were pooled and sequenced in two lanes of an Illumina Hiseq 2500 instrument at the Clinical Genomics Centre in Mount Sinai Hospital and TruSight libraries were pooled in groups of three and sequenced in an Illumina MiSeq at our institutional genomics core. Data analysis was performed using the CLC Genomics Workbench v7.0 software by mapping the reads to the reference Human genome hg19 (GRCh37 Ensemble 74). Single nucleotide variants were identified using a quality based variant detection with a minimum coverage cutoff of $10 \times$ and a minimum frequency of $35 \%$ for heterozygous alleles. Variants were annotated using the dbSNP database (http://www.ncbi.nlm.nih.gov/snp/). Only SNPs identified in the target regions and in all three active TB cases, and absent in the two latent TB cases, were kept for analysis.

\section{Outcomes}

The total number of targets covered with at least a $10 \times$ coverage ranged from 94.6 to $96 \%$ for the SureSelect and $93.8-94.9 \%$ for the TruSight kit. The two enrichment kits performed relatively similar in terms of time and cost of library preparation and sequencing costs. Promoter regions located outside of the untranslated regions (UTRs) are not covered in any of the two kits and therefore potential polymorphisms in these regions are not identified. However, given the large number of exon regions covered by the SureSelect, this kit is ideal to use in a discovery assay for the identification of novel polymorphisms associated to a given condition.

Using the SureSelect kit we identified 1611 SNPs that were only present in the Active TB cases. Using the TruSight kit we identified 182 SNPs only present in the active TB cases and not in the latent TB participants. The overlap of the two kits was 112 SNPs (Additional file 1: Table S1). The 70 SNPs found in the TruSight kit data that did not overlap with the SureSelect kit were examined in more detail. Of these, 21 were in target regions not covered by the SureSelect kit, 15 were not called in the SureSelect data because the ratio of alleles was $<35 \%, 26$ were in found in the latent cases but not called in the TruSight data due to insufficient coverage, six were not called in the SureSelect data due to insufficient coverage and two were incorrect calling due to stretches of homopolymers.

Since TB is an infectious disease with a complex interaction between host, pathogen and environmental factors [23], it is possible that multiple genetic variants in the host may be involved in susceptibility to disease as opposed to rare genetic disorders that are often explained by single variants [1]. A gene ontology (GO) enrichment analysis was performed in order to identify possible pathways, as opposed to single variants, that may be involved in susceptibility to TB. When the 1611 SNPs identified in active TB cases only using the SureSelect kit were analyzed, several molecular pathways were identified as being significantly enriched ( $p$ value $<0.01$ ), including some with a known link to TB infection. These included (1) cellular response to ATP and purinergic nucleotide receptor signaling pathway; (2) apoptotic processes; and (3) vitamin metabolic and biosynthetic processes (Table 1). These pathways were also identified when only the 112 SNPs that overlap with the two kits were analyzed (data not shown).

We also interrogated our data against a previously reported list of variants in 26 innate immune genes that have been shown to be associated with susceptibility to TB [4]. For this analysis we used the entire SSTE SNP data set that the met the quality standards outlined above. We included variants identified in intron or intergenic regions only if sufficient coverage $(10 \times)$ was 
Table 1 Gene ontology (GO) enrichment analysis of 1611 SNPs identified at 10x coverage using SureSelect kit with $P<0.01$

\begin{tabular}{|c|c|c|c|c|}
\hline \multirow[t]{2}{*}{ GO description } & \multicolumn{2}{|c|}{ Occurrences } & \multirow[t]{2}{*}{ P values } & \multirow[t]{2}{*}{ Link to tuberculosis } \\
\hline & All genes & Sample & & \\
\hline Cellular response to ATP & 7 & 4 & $3.23 \mathrm{E}-4$ & {$[41,42]$} \\
\hline Riboflavin metabolic process & 5 & 3 & $1.71 \mathrm{E}-3$ & \\
\hline Regulation of centriole replication & 5 & 3 & $1.71 \mathrm{E}-3$ & \\
\hline Axon guidance & 281 & 28 & $2.98 \mathrm{E}-3$ & \\
\hline Vitamin E metabolic process & 2 & 2 & $3.27 \mathrm{E}-3$ & {$[43]$} \\
\hline Epithelial cell proliferation & 2 & 2 & $3.27 \mathrm{E}-3$ & \\
\hline Response to cortisol stimulus & 2 & 2 & $3.27 \mathrm{E}-3$ & \\
\hline Negative regulation of retinal ganglion cell axon guidance & 2 & 2 & $3.27 \mathrm{E}-3$ & \\
\hline Cellular response to hormone stimulus & 12 & 4 & $3.63 \mathrm{E}-3$ & \\
\hline O-glycan processing & 55 & 9 & $3.71 \mathrm{E}-3$ & \\
\hline Regulation of small GTPase mediated signal transduction & 121 & 15 & $3.75 \mathrm{E}-3$ & \\
\hline Integrin-mediated signaling pathway & 37 & 7 & $4.45 E-3$ & \\
\hline Purinergic nucleotide receptor signaling pathway & 7 & 3 & $5.49 \mathrm{E}-3$ & {$[44]$} \\
\hline Synaptic vesicle endocytosis & 7 & 3 & $5.49 E-3$ & \\
\hline Negative regulation of phosphatase activity & 49 & 8 & $6.18 \mathrm{E}-3$ & \\
\hline Response to unfolded protein & 30 & 6 & $6.26 \mathrm{E}-3$ & {$[45]$} \\
\hline Autophagic vacuole fusion & 8 & 3 & $8.41 \mathrm{E}-3$ & {$[46]$} \\
\hline Nucleocytoplasmic transport & 15 & 4 & $8.73 E-3$ & \\
\hline Small GTPase mediated signal transduction & 158 & 17 & $8.93 \mathrm{E}-3$ & \\
\hline Pre-B cell allelic exclusion & 3 & 2 & $9.43 \mathrm{E}-3$ & \\
\hline Pressure natriuresis & 3 & 2 & $9.43 \mathrm{E}-3$ & \\
\hline Chemorepulsion involved in embryonic olfactory bulb interneuron precursor migration & 3 & 2 & $9.43 \mathrm{E}-3$ & \\
\hline Positive regulation of telomere maintenance via telomerase & 3 & 2 & $9.43 \mathrm{E}-3$ & \\
\hline Lacrimal gland development & 3 & 2 & $9.43 \mathrm{E}-3$ & \\
\hline Roundabout signaling pathway & 3 & 2 & $9.43 \mathrm{E}-3$ & \\
\hline Leukotriene B4 catabolic process & 3 & 2 & $9.43 E-3$ & \\
\hline Vitamin K biosynthetic process & 3 & 2 & $9.43 E-3$ & \\
\hline Metaphase plate congression & 3 & 2 & $9.43 \mathrm{E}-3$ & \\
\hline Regulation of endopeptidase activity & 3 & 2 & $9.43 \mathrm{E}-3$ & \\
\hline Apoptotic process involved in luteolysis & 3 & 2 & $9.43 \mathrm{E}-3$ & \\
\hline
\end{tabular}

present in all five samples. We identified SNPs in several innate immune genes previously shown to be potentially associated with active TB [4] (Table 2). Based on the SNPs identified in active TB subjects, three of these genes appear to be related to active TB in our study (i.e. TLR1, TNF and VDR). Although we identified SNPs in the remaining genes listed in Table 2, these were found in both latent and active TB cases or only in individuals with latent TB. Of the three genes with SNPs present in active TB cases but not in latent cases: toll-like receptor-1 (TLR1), vitamin D receptor (VDR) and tumor necrosis factor (TNF), only SNPs in TLR1 were found in all three active cases. SNPs in these three genes were also confirmed by Sanger sequencing (Additional file 2). Both the A743G (Asn148Ser) and G1805T (Ser602Ile) variants were identified in TLR1. Although data regarding a link between TLR1 variants and susceptibility to TB is ambiguous [24-27], it has been shown that the 602Ser allele inhibits cell surface trafficking leading to a lack of TLR1 on the plasma membrane and a hypo-responsiveness to TLR1 agonists including mycobacterial membrane preparations $[24,25,28]$. The role of VDR and susceptibility to TB is also under debate $[29,30]$ but the active metabolite of vitamin D (1,25-dihydroxyvitamin D3) suppresses the growth of $M$. tuberculosis in vitro [31,32] and vitamin D deficiency has been associated with susceptibility to active TB [33]. TNF is a cytokine that plays a key role in granuloma formation [34-36]. Neutralization of TNF leads to a lack of control of initial or chronic infection and loss of granuloma structure. There have been 
Table 2 Details of SNPs found in innate immune genes at 10x coverage using SSTE kit which have been associated with susceptibility to TB [4]

\begin{tabular}{|c|c|c|c|c|c|c|}
\hline Gene & Polymorphism SNP & Coding change & Amino acid change & Genetic location & Active cases & Latent cases \\
\hline \multirow[t]{2}{*}{ TLR1 } & rs4833095 & $743 A>G$ & Asn248Ser & Exon & 3 & 0 \\
\hline & rs5743618 & $1805 G>T$ & Ser602lle & Exon & 3 & 0 \\
\hline TLR8 & rs3764880 & $1 A>G$ & Met1Val & & 2 & 1 \\
\hline TLR9 & rs352143 & $169 A>G$ & Asn57Asp & & 1 & 1 \\
\hline TIRAP & rs8177374 & $539 \mathrm{C}>\mathrm{T}$ & Ser180Leu & & 0 & 1 \\
\hline \multirow[t]{2}{*}{ NOD2 } & rs2066842 & $802 \mathrm{C}>\mathrm{T}$ & Pro268Ser & Exon & 0 & 1 \\
\hline & rs2066844 & $2104 C>T$ & Arg702Trp & Exon & 0 & 1 \\
\hline \multirow[t]{2}{*}{ VDR } & rs2228570 & $2 \mathrm{~T}>\mathrm{C}$ & Met1 & & 3 & 2 \\
\hline & rs731236 (Taql) & $1056 \mathrm{~T}>\mathrm{C}$ & & Coding-synon & 1 & 0 \\
\hline ILIB & rs1143634 & $315 \mathrm{C}>\mathrm{T}$ & & Coding-synon & 0 & 1 \\
\hline TNF & rs1800629 & $-308 \mathrm{G} / \mathrm{A}$ & & Promoter & 1 & 0 \\
\hline
\end{tabular}

Multiple SNPs that have not been previously reported to be associated with susceptibility to TB were found in all of these genes

multiple conflicting studies examining the role of SNPs in the TNF promoter at the -308 position and TB susceptibility [37-40] however there is no evidence that SNPs in this region effect the transcription of TNF [36]. Conflicting results is not surprising in a complex diseases like TB, where many gene variants could be interacting to result in disease, and the combination of different gene variants could lead to the same outcome.

\section{Impacts}

The work outlined in this study was completed in 16 months. This included review by the Ethics Board, training of the research nurse to recruit potential participants in one of Toronto's under-housed shelters, recruitment, sample collection, sequencing and data analysis. Active recruitment of participants at the shelter site was completed in only 1 month. However, even in that short period of time we were able to recruit nine potential participants and we anticipate that larger trials with an expansion in duration of recruitment as well as number of targeted shelters will result in a much larger participation by individuals that meet the recruitment specifications. Even though our pilot study only included three individuals with a history of active TB and two individuals with confirmed TB infection but not disease, exome sequencing proved to be a powerful technique to identify potential host genetic variants associated with susceptibility to TB disease. Future studies expanding the number of individuals in each cohort will help narrow down the number of potential targets for validation.

To our knowledge, this is the first study that has used exome sequencing to potentially identify genomic markers of susceptibility to TB resulting in a large set of data including potential single nucleotide variants as well as molecular and cellular pathways that may be associated with TB infection and disease.

\section{Additional files}

Additional file 1. Materials and methods for PCR and Sanger sequencing of selected SNPS.

Additional file 2. List of SNPs identified using both sequencing kits (overlapping SNPS)

\section{Authors' contributions}

CD carried out DNA extraction and preparation for sequencing, data analysis, interpretation of data and manuscript drafting. FJ participated in the design of the study and helped draft the manuscript. CM conceived of the study, participated in study design and coordination and helped to draft the manuscript. All authors read and approved the final manuscript.

\section{Author details}

1 Public Health Ontario, Toronto, ON, Canada. ${ }^{2}$ Department of Laboratory Medicine and Pathobiology, University of Toronto, Toronto, ON, Canada.

${ }^{3}$ Department of Microbiology, Immunology and Pathology, Colorado State University, 1619 Campus Delivery, Fort Collins, CO 80523-1601, USA.

\section{Acknowledgements}

We thank Julianne for her involvement recruiting participants and performing sample collection for the study. We are very thankful to all the participants in the study. Without them this study would have not been possible. This study was funded by the McLaughlin Centre Accelerator Grant \#MC-2013-09 to CM (PI).

\section{Competing interests}

The authors declare that they have no competing interests.

Received: 25 August 2015 Accepted: 25 November 2015 Published online: 08 December 2015

\section{References}

1. Daya M, van der Merwe L, van Helden PD, Moller M, Hoal EG. Investigating the role of gene-gene interactions in TB susceptibility. PLoS One. 2015;10(4):e0123970.

2. Moller M, Hoal EG. Current findings, challenges and novel approaches in human genetic susceptibility to tuberculosis. Tuberculosis (Edinb). 2010;90(2):71-83.

3. Stein CM, Zalwango S, Malone LL, Won S, Mayanja-Kizza H, Mugerwa RD et al. Genome scan of M. tuberculosis infection and disease in Ugandans. PLoS One. 2008;3(12):e4094. 
4. Azad AK, Sadee W, Schlesinger LS. Innate immune gene polymorphisms in tuberculosis. Infect Immun. 2012;80(10):3343-59.

5. Bulat-Kardum LJ, Etokebe GE, Lederer P, Balen S, Dembic Z. Genetic polymorphisms in the toll-like receptor 10, interleukin (IL)17A and IL17F genes differently affect the risk for tuberculosis in Croatian population. Scand J Immunol. 2015;82(1):63-9. doi:10.1111/sji.12300.

6. Chen Z, Wang W, Liang J, Wang J, Feng S, Zhang G. Association between toll-like receptors 9 (TLR9) gene polymorphism and risk of pulmonary tuberculosis: meta-analysis. BMC Pulm Med. 2015;15(1):57.

7. Hu Y, Wu L, Li D, Zhao Q, Jiang W, Xu B. Association between cytokine gene polymorphisms and tuberculosis in a Chinese population in Shanghai: a case-control study. BMC Immunol. 2015;16:8-015-0071-6.

8. Shi GL, Yang L, Sun Y, Yin YJ, Song CX. MCP-1 gene polymorphisms in North Chinese patients with pulmonary tuberculosis. Genet Mol Res. 2015;14(2):4035-40.

9. Zhang $Y$, Jiang $T$, Yang $X$, Xue $Y$, Wang $C$, et al. Toll-like receptor-1, - 2, and -6 polymorphisms and pulmonary tuberculosis susceptibility: a systematic review and meta-analysis. PLoS One. 2013;8(5):e63357.

10. Davila S, Hibberd ML, Hari Dass R, Wong HE, Sahiratmadja E, Bonnard C, et al. Genetic association and expression studies indicate a role of toll-like receptor 8 in pulmonary tuberculosis. PLoS Genet. 2008;4(10):e1000218.

11. Hansen MF, Johansen J, Bjørnevoll I, Sylvander AE, Steinsbekk KS, Sætrom P, Sandvik AK, Drabløs F, Sjursen W. A novel POLE mutation associated with cancers of colon, pancreas, ovaries and small intestine. Fam Cancer. 2015;14(3):437-48. doi:10.1007/s10689-015-9803-2.

12. Jiao H, Arner P, Gerdhem P, Strawbridge RJ, Näslund E, Thorell A, Hamsten A, Kere J, Dahlman I. Exome sequencing followed by genotyping suggests SYPL2 as a susceptibility gene for morbid obesity. Eur J Hum Genet. 2015;23(9):1216-22. doi:10.1038/ejhg.2014.255.

13. Shortt K, Chaudhary S, Grigoryev D, Heruth DP, Venkitachalam L, Zhang LQ, Ye SQ. Identification of novel single nucleotide polymorphisms associated with acute respiratory distress syndrome by exome-seq. PLoS One. 2014;9(11):e111953.

14. Liu L, Okada S, Kong XF, Kreins AY, Cypowyj S, Abhyankar A, et al. Gainof-function human STAT1 mutations impair IL-17 immunity and underlie chronic mucocutaneous candidiasis. J Exp Med. 2011;208(8):1635-48.

15. Kong XF, Bousfiha A, Rouissi A, Itan Y, Abhyankar A, Bryant V, et al. A novel homozygous p. R1105X mutation of the AP4E1 gene in twins with hereditary spastic paraplegia and mycobacterial disease. PLoS One. 2013;8(3):e58286

16. Manry J, Quintana-Murci L. A genome-wide perspective of human diversity and its implications in infectious disease. Cold Spring Harb Perspect Med. 2013;3(1):a012450.

17. Brites D, Gagneux S. Co-evolution of Mycobacterium tuberculosis and Homo sapiens. Immunol Rev. 2015;264(1):6-24.

18. Spigelman M, Donoghue HD, Abdeen Z, Ereqat S, Sarie I, Greenblatt CL, Pap I, Szikossy I, Hershkovitz I, Bar-Gal GK, Matheson C. Evolutionary changes in the genome of Mycobacterium tuberculosis and the human genome from 9000 years BP until modern times. Tuberculosis (Edinb). 2015;95(Suppl 1):S145-9. doi:10.1016/j.tube.2015.02.022.

19. Alexander DC, Guthrie JL, Pyskir D, Maki A, Kurepina N, Kreiswirth BN, et al. Mycobacterium tuberculosis in Ontario, Canada: insights from IS6110 restriction fragment length polymorphism and mycobacterial interspersed repetitive-unit-variable-number tandem-repeat genotyping. J Clin Microbiol. 2009:47(8):2651-4

20. Khan K, Rea E, McDermaid C, Stuart R, Chambers C, Wang J, et al. Active tuberculosis among homeless persons, Toronto, Ontario, Canada, 1998-2007. Emerg Infect Dis. 2011;17(3):357-65.

21. Mehaffy C, Guthrie JL, Alexander DC, Stuart R, Rea E, Jamieson FB. Marked microevolution of a unique Mycobacterium tuberculosis strain in 17 years of ongoing transmission in a high risk population. PLoS One. 2014;9(11):e112928.

22. Adam HJ, Guthrie JL, Bolotin S, Alexander DC, Stuart R, Pyskir D, et al. Genotypic characterization of tuberculosis transmission within Toronto's under-housed population, 1997-2008. Int J Tuberc Lung Dis. 2010;14(10):1350-3

23. Comas I, Gagneux S. The past and future of tuberculosis research. PLoS Pathog. 2009;5(10):e1000600.

24. Hart BE, Tapping RI. Differential trafficking of TLR1 I602S underlies host protection against pathogenic mycobacteria. J Immunol. 2012;189(11):5347-55.
25. Johnson CM, Lyle EA, Omueti KO, Stepensky VA, Yegin O, Alpsoy E, et al. Cutting edge: a common polymorphism impairs cell surface trafficking and functional responses of TLR1 but protects against leprosy. J Immunol. 2007;178(12):7520-4

26. Ma X, Liu Y, Gowen BB, Graviss EA, Clark AG, Musser JM. Full-exon resequencing reveals toll-like receptor variants contribute to human susceptibility to tuberculosis disease. PLoS One. 2007;2(12):e1318.

27. Uciechowski P, Imhoff H, Lange C, Meyer CG, Browne EN, Kirsten DK, et al. Susceptibility to tuberculosis is associated with TLR1 polymorphisms resulting in a lack of TLR1 cell surface expression. J Leukoc Biol. 2011;90(2):377-88.

28. Hawn TR, Misch EA, Dunstan SJ, Thwaites GE, Lan NT, Quy HT, et al. A common human TLR1 polymorphism regulates the innate immune response to lipopeptides. Eur J Immunol. 2007;37(8):2280-9.

29. Bornman L, Campbell SJ, Fielding K, Bah B, Sillah J, Gustafson P, et al. Vitamin D receptor polymorphisms and susceptibility to tuberculosis in West Africa: a case-control and family study. J Infect Dis. 2004;190(9):1631-41.

30. Bellamy R, Ruwende C, Corrah T, McAdam KP, Thursz M, Whittle HC, et al. Tuberculosis and chronic hepatitis B virus infection in Africans and variation in the vitamin D receptor gene. J Infect Dis. 1999;179(3):721-4.

31. Rockett KA, Brookes R, Udalova I, Vidal V, Hill AV, Kwiatkowski D. 1,25-Dihydroxyvitamin D3 induces nitric oxide synthase and suppresses growth of Mycobacterium tuberculosis in a human macrophage-like cell line. Infect Immun. 1998;66(11):5314-21.

32. Denis M. Killing of Mycobacterium tuberculosis within human monocytes: activation by cytokines and calcitriol. Clin Exp Immunol. 1991;84(2):200-6.

33. Wilkinson RJ, Llewelyn M, Toossi Z, Patel P, Pasvol G, Lalvani A, et al. Influence of vitamin $D$ deficiency and vitamin $D$ receptor polymorphisms on tuberculosis among Gujarati Asians in west London: a case-control study. Lancet. 2000;355(9204):618-21.

34. Algood HM, Lin PL, Yankura D, Jones A, Chan J, Flynn JL. TNF influences chemokine expression of macrophages in vitro and that of CD11b+ cells in vivo during Mycobacterium tuberculosis infection. J Immunol. 2004;172(11):6846-57

35. Mohan VP, Scanga CA, Yu K, Scott HM, Tanaka KE, Tsang E, et al. Effects of tumor necrosis factor alpha on host immune response in chronic persistent tuberculosis: possible role for limiting pathology. Infect Immun. 2001;69(3):1847-55.

36. Bayley JP, Ottenhoff TH, Verweij CL. Is there a future for TNF promoter polymorphisms? Genes Immun. 2004;5(5):315-29.

37. Correa PA, Gomez LM, Cadena J, Anaya JM. Autoimmunity and tuberculosis. Opposite association with TNF polymorphism. J Rheumatol. 2005;32(2):219-24.

38. Hu Y, Wu L, Li D, Zhao Q, Jiang W, Xu B. Association between cytokine gene polymorphisms and tuberculosis in a Chinese population in Shanghai: a case-control study. BMC Immunol. 2015;16:8-015-0071-6.

39. Merza M, Farnia P, Anoosheh S, Varahram M, Kazampour M, Pajand O, et al. The NRAMPI, VDR and TNF-alpha gene polymorphisms in Iranian tuberculosis patients: the study on host susceptibility. Braz J Infect Dis. 2009;13(4):252-6

40. Sharma S, Rathored J, Ghosh B, Sharma SK. Genetic polymorphisms in TNF genes and tuberculosis in North Indians. BMC Infect Dis. 2010;10:165-2334-10-165.

41. Ramachandra L, Qu Y, Wang Y, Lewis CJ, Cobb BA, Takatsu K, et al. Mycobacterium tuberculosis synergizes with ATP to induce release of microvesicles and exosomes containing major histocompatibility complex class II molecules capable of antigen presentation. Infect Immun. 2010;78(12):5116-25.

42. Koul A, Vranckx L, Dhar N, Gohlmann HW, Ozdemir E, Neefs JM, et al. Delayed bactericidal response of Mycobacterium tuberculosis to bedaquiline involves remodelling of bacterial metabolism. Nat Commun. 2014;5:3369.

43. Gupta KB, Gupta R, Atreja A, Verma M, Vishvkarma S. Tuberculosis and nutrition. Lung India. 2009;26(1):9-16.

44. Miller CM, Boulter NR, Fuller SJ, Zakrzewski AM, Lees MP, Saunders BM, et al. The role of the P2X(7) receptor in infectious diseases. PLoS Pathog. 2011;7(11):e1002212.

45. Seimon TA, Kim MJ, Blumenthal A, Koo J, Ehrt S, Wainwright H, et al. Induction of ER stress in macrophages of tuberculosis granulomas. PLoS One. 2010;5(9):e12772.

46. Pacheco SA, Powers KM, Engelmann F, Messaoudi I, Purdy GE. Autophagic killing effects against by alveolar macrophages from young and aged Rhesus macaques. PLoS One. 2013:8(6):e66985. 